\title{
A True Full-Duplex IO (TFD-IO) with Background SI Cancellation for High-Density Interfaces
}

\author{
Sandeep Goyal, Ganpat Parulekar, and Shalabh Gupta
}

\begin{abstract}
In this work, we have proposed and experimentally demonstrated a true full-duplex IO (TFD-IO) for high-speed high-density interfaces. The proposed TFD-IO can be used as an independent module that converts a unidirectional IO/interconnect to a fully bidirectional IO/interconnect, to ideally double the throughput of the high-speed interface. The TFD-IO uses a correlation-based technique to cancel the self-interference (SI) adaptively in the background. The signals transmitted from the near-end and the far-end can use independent baud-rates and signaling schemes in a TFD-IO. A proof-of-concept design of the TFD-IO module has been fabricated in a $65 \mathrm{~nm}$ CMOS technology, and demonstrated with bidirectional throughputs of up to $12.8 \mathrm{~Gb} / \mathrm{s}$.
\end{abstract}

Index Terms-Adaptive echo cancellation (EC), chiplets, full-Duplex, high-speed serial links, heterogeneous integration, source-synchronous transceiver, simultaneous bidirectional (SBD).

\section{INTRODUCTION}

Recent growth in the requirements and capabilities of algorithms for machine learning and artificial intelligence based user applications has led to a huge demand for improved performance of computing systems. These systems need to store, process, and exchange large amounts of data to meet the growing demands. In these systems, data interfaces, such as processor-to-processor, processor-to-memories and processorto-network interfaces, may have to handle data throughputs of several terabits/second and interface densities of greater than terabits/second/mm [1], [2]. Throughputs of these high-speed interfaces are limited by interconnect bandwidths and chip packaging technologies [3], [4]. Multi-chip modules (MCM), system-in-package ( $\mathrm{SiP})$, and multi-chiplet based platforms with die-to-die interfaces have emerged as solutions to overcome some of the limitations [4]-[7]. The die-to-die interfaces need to support high data/pin efficiencies, low channel losses, and small form-factors [4], [5], [8].

The interface with a large number of lanes suffers from the issue of crosstalk between them, which further deteriorates the performance with an increase in the lane density or baud-rate [9], [10]. Reduction in the number of lanes is possible by using simultaneous bidirectional (SBD) or full-duplex (FD) communication between the high-performance modules. FD links use transceivers at the two ends, which are named as far-end and near-end. The strength of the near-end transmitted signal at the input-output (IO) port in the full-duplex link is much higher than that of the signal received from the far-end. Thus, the near-end transmitter causes a severe self-interference

The authors are with the Department of Electrical Engineering, Indian Institute of Technology Bombay, Mumbai - 400076, India (email: sandeepgoyal@ee.iitb.ac.in; ganpatp@ee.iitb.ac.in; shalabh@ee.iitb.ac.in).

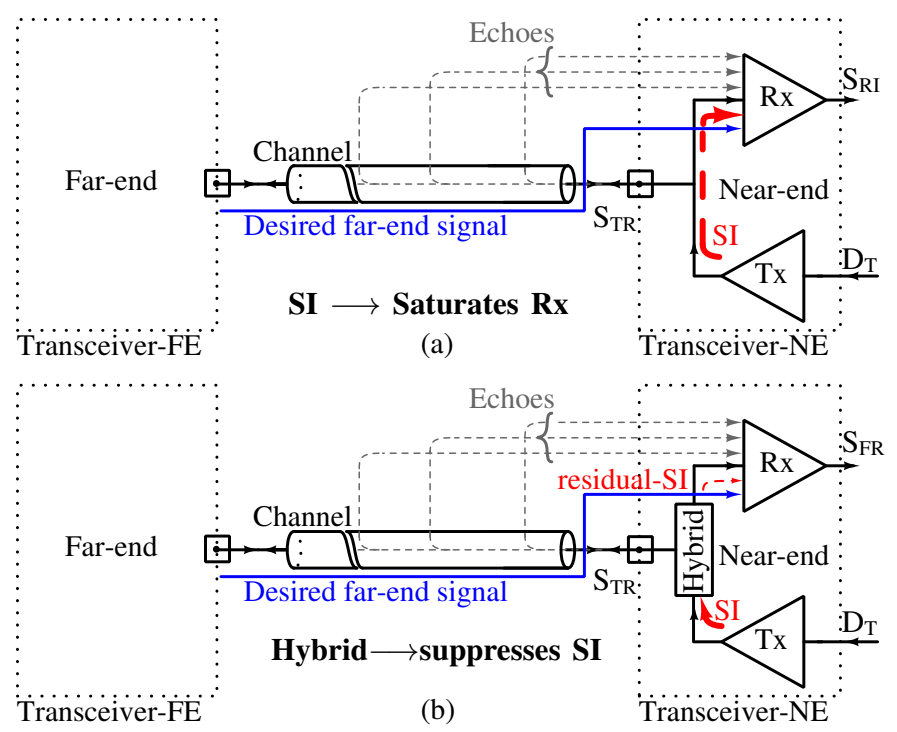

Fig. 1: Conventional bidirectional links: (a) without interference cancellation and (b) with the hybrid used for suppressing the SI. Various constituents of near-end interference, i.e., SI and echoes, are shown using dashed lines. Here, Tx - transmitter; $\mathrm{Rx}$ : receiver; $\mathrm{D}_{\mathrm{T}}$ : data to be transmitted; SI : self-interference; $\mathrm{S}_{\mathrm{TR}}$ : composite signal at transceiver IO port; $\mathrm{S}_{\mathrm{RI}}$ : signal received with $\mathrm{SI}$; and $\mathrm{S}_{\mathrm{FR}}$ : recovered far-end signal.

(SI) at the near-end receiver. In addition, there can be echoes of the transmitted signal at the receiver due to a mismatch between the source and load impedances, and back reflections from the channel. Therefore, transceivers in these links receive a composite signal $S_{\mathrm{RI}}$, which consists of interference from the signal transmitted by the near-end transceiver along with the desired far-end signal, as shown in Fig. 1(a).

The interference hinders recovery of the far-end information at the near-end receiver. As a result, along with a data transmission mechanism, the transceivers also need a method to suppress a significant part of the SI at the receiver front-end itself, before the received signal can be further processed to recover the data transmitted from the far-end. For example, as shown in Fig. 1(b), a hybrid can be used to suppress the SI to residual-SI, which may be cancelled in the digital domain along with the echoes.

Full-duplex links for Ethernet standards, such as IEEE 802.3ak and 802.3ap, typically use passive hybrids and an auxiliary digital-to-analog-converter (DAC) for interference suppression in the analog domain [11]-[15], before the received signal is further processed for data recovery. The passive hybrids are magnetic, bulky, and band-limiting in nature. They support suppression only at low baud-rates, making them 


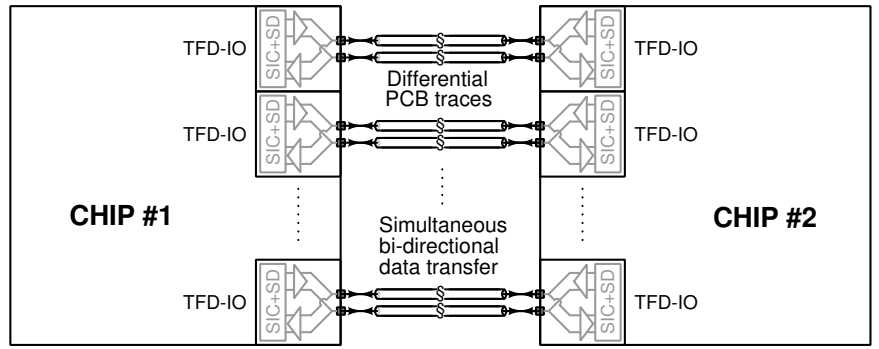

Fig. 2: Bidirectional chip-to-chip data transfer interface with the proposed true full-duplex IOs (TFD-IO)s.

unattractive for high-density and high-speed interconnects because of their larger form factors. To achieve higher throughputs, the high-speed FD interconnects proposed/demonstrated recently have used hybrids with active circuits for interference suppression [15]-[28]. These hybrids effectively consist of one of the following: (i) a scaled replica generator with a subtractor [11]-[13], [21], [28]; (ii) a comparator with dynamic referencing [16], [20], [23]-[27]; (iii) a resistive or a capacitive bridge [21], [22]; (iv) a resistor-transconductor (R-gm) cell [17], [19]; and (v) a directional inverter/buffer (DIB) with weighted cancellation paths [29]. The main limitation in these schemes is that they do not account for the delay spread of the transmitted pulses that causes SI over multiple bit periods. These hybrids may also require manual tuning or foreground calibration to support data transfer over a wide range of interface conditions. Furthermore, most of these techniques require timing synchronization between the far-end and the near-end signals, to ensure that the interference is suppressed/cancelled sufficiently, as the amount of cancellation depends on the receiver sampling phase [19], [20], [30], [31].

To overcome the above mentioned limitations, digital techniques have to be implemented, which can support interference cancellation adaptively [18]. However, implementing SI cancellation using digital approaches is often power and area inefficient. Furthermore, a significant training time may be required for adaption of the SI-canceller's weight coefficients using the digital techniques. In this paper, we propose a true full-duplex IO (TFD-IO) module that supports simultaneous bidirectional data transmission over an electrical interconnect. The TFD-IO uses a correlation-based SI-canceller, whose weight coefficients are adjusted in the background, adaptively, using a modulation format and data-rate independent technique [32], [33]. Fig. 2 shows a conceptual diagram of a high-density chip-to-chip interface, wherein, the TFD-IOs can be used as plug-n-play modules to double the data efficiency per lane and per IO port of the chip. The use of the bidirectional ports, i.e. the TFD-IOs, can also simplify board level design while interfacing two chips on a PCB with a large number of highbandwidth interconnects between them.

In the following section, we review the correlation-based interference cancellation technique. In Section III, we present the proposed TFD-IO and its implementation details. Section IV presents the post-layout simulation results obtained for the TFD-IO cells. Section V presents the measurement setup and results obtained for the TFD-IO cells, and Section VI concludes this brief.

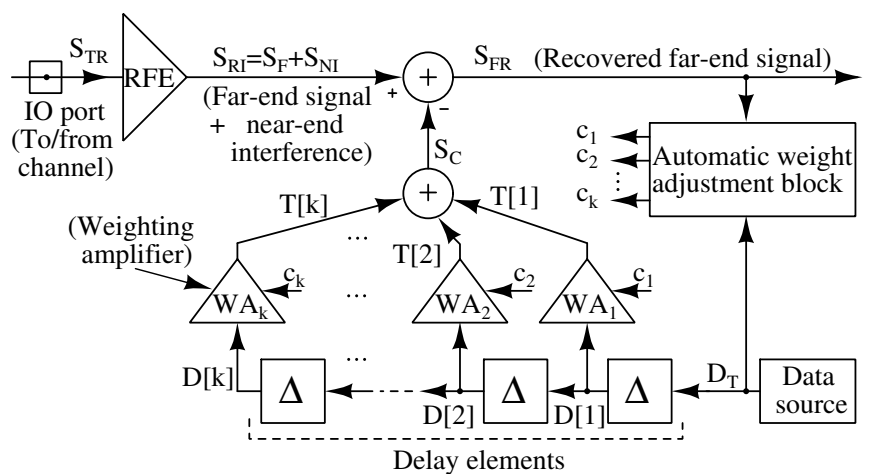

Fig. 3: System model for a correlation-based interference canceller. Here, $\mathrm{D}_{\mathrm{T}}$ : Data to be transmitted; $\mathrm{D}[\mathrm{k}]: \mathrm{k}^{\text {th }}$ delayed tap $\mathrm{D}_{\mathrm{T}} ; \mathrm{T}[\mathrm{k}]: \mathrm{k}^{\text {th }}$ weighted tap; $c_{k}: k^{\text {th }}$ weight coefficient; $\mathrm{WA}_{\mathrm{k}}: \mathrm{k}^{\text {th }}$ weighting amplifier (WA); $\mathrm{S}_{\mathrm{TR}}$ : composite signal at transceiver IO port; $\mathrm{S}_{\mathrm{RI}}$ : signal received with $\mathrm{SI} ; \mathrm{S}_{\mathrm{F}}$ : far-end signal; $\mathrm{S}_{\mathrm{NI}}$ : near-end interference; and $\mathrm{S}_{\mathrm{FR}}$ : recovered far-end signal.

\section{CORRELATION-BASED SI CANCELLATION}

The near-end transceiver in a FD link receives a composite signal $S_{R I}$, which contains: (i) the far-end signal $S_{F}$; and (ii) $\mathrm{S}_{\mathrm{NI}}$ - includes the SI and echoes. The major challenge in transferring data over FD links is to extract the desired farend information from $S_{\mathrm{RI}}$. This extraction becomes further challenging when the near-end transceiver should suppress the interference unconscious of the far-end transceiver. The used correlation-based cancellation approach allows for such an interference suppression, independent of the operating state of the far-end transceiver. This approach uses a practical assumption that the data signals transmitted by two independent data sources are typically uncorrelated. For such uncorrelated signals, this cancellation approach allows the SI-canceller to suppress the interference by adapting its coefficients in the background. This approach thus eliminates the need of training the SI-canceller in foreground, which is required to track the variations in data transmission path.

Figure 3 illustrates the model of a transceiver with a correlation-based cancellation loop used for suppressing the near-end interference. The cancellation approach uses a simple fact that the two signals, $\mathrm{D}_{\mathrm{T}}$ and $\mathrm{S}_{\mathrm{NI}}$, have the same origin, and hence are correlated. In this approach, to suppress SI, the cancellation loop minimizes only the correlation present between output of the canceller $\mathrm{S}_{\mathrm{FR}}$ (the recovered far-end signal) and $\mathrm{D}[1: \mathrm{k}]$ - a set of delayed taps (copies) of $\mathrm{D}_{\mathrm{T}}$. These delayed taps are required to mimic the delay spreads and magnitude responses of the interference path. The cancellation loop, thus extracts the uncorrelated far-end information from the $S_{R I}$. For SI cancellation, the delayed taps $D[1: k]$ are first weighted with the corresponding weight coefficients $\mathrm{c}_{[1: \mathrm{k}]}$, and are then summed together to generate a replica cancellation signal $\mathrm{S}_{\mathrm{C}}$. Next, $\mathrm{S}_{\mathrm{C}}$ is subtracted from $\mathrm{S}_{\mathrm{RI}}$ to minimize $\mathrm{SI}$, as given by Eq. (3).

$$
\begin{aligned}
\mathrm{S}_{\mathrm{FR}} & =\mathrm{S}_{\mathrm{RI}}-\mathrm{S}_{\mathrm{C}}, \\
& =\mathrm{S}_{\mathrm{F}}+\mathrm{SI}-\sum_{\mathrm{p}=1}^{\mathrm{k}} \mathrm{c}_{\mathrm{p}} \times \mathrm{D}[\mathrm{p}],
\end{aligned}
$$




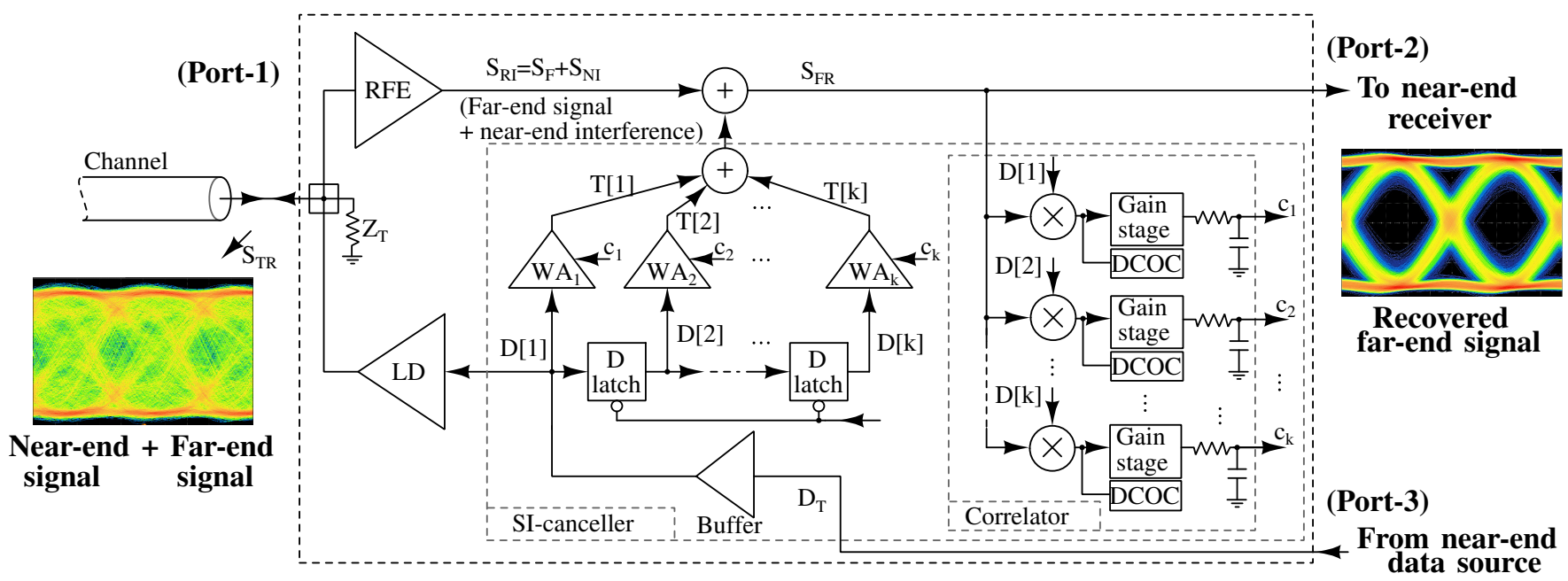

Fig. 4: Proposed true full-duplex IO with a correlation-based SI-canceller. Here, DCOC: DC offset compensator.

where, $\mathrm{D}[\mathrm{p}]$ denotes the $\mathrm{p}^{\text {th }}$ delayed tap of $\mathrm{D}_{\mathrm{T}}$, and $\mathrm{c}_{\mathrm{p}}$ is the weight coefficient (gain) of weighting amplifier $\mathrm{WA}_{\mathrm{p}}$. To extract the far-end information by suppressing the interference, i.e., to make $\mathrm{S}_{\mathrm{FR}}$ free of SI or of any interfering component due to the delayed taps $\mathrm{D}[1: \mathrm{k}]$, Eq. (2) must be satisfied.

$$
\mathrm{SI}=\sum_{\mathrm{p}=1}^{\mathrm{k}} \mathrm{G}_{\text {Loop }} \times \mathrm{R}_{\mathrm{p}} \times \mathrm{D}[\mathrm{p}],
$$

where, $R_{p}$ denotes the correlation present between $S_{F R}$ and $\mathrm{D}[\mathrm{p}]$, and $\mathrm{G}_{\text {Loop }}$ denotes the loop gain of the cancellation loop. Let us consider for a delayed tap $\mathrm{D}[\mathrm{p}], \varepsilon_{\mathrm{p}}$ is the desired tap weight to suppress the amount of interference present in the $\mathrm{S}_{\mathrm{RI}}$. Therefore, for the cancellation, $\varepsilon_{\mathrm{p}}$ is the required value for $c_{p}$, and it can be determined as per the following Equation,

$$
\varepsilon_{\mathrm{p}} \times \mathrm{D}[\mathrm{p}]=\mathrm{G}_{\text {Loop }} \times \mathrm{R}_{\mathrm{p}} \times \mathrm{D}[\mathrm{p}],
$$

where, $R_{p}$ for a system in its linear range of operation can be further divided into three parts as given by the following Equation,

$$
\mathrm{R}_{\mathrm{p}}=\mathrm{R}_{\mathrm{PI}}+\mathrm{R}_{\mathrm{PF}}+\mathrm{R}_{\mathrm{PC}}
$$

where, $\mathrm{R}_{\mathrm{PI}}$ denotes the correlation between $\mathrm{D}[\mathrm{p}]$ and $\mathrm{SI}$, $R_{P F}$ denotes the correlation between $D[p]$ and $S_{F}$, and $R_{P C}$ denotes the correlation between $\mathrm{D}[\mathrm{p}]$ and $\mathrm{S}_{\mathrm{C}}$. For interference cancellation, the used correlation-based approach supports minimization of these correlation components as follows:

- $R_{P F}$ : should be equal to zero as the two transmitted signals, i.e., the near-end signal and the far-end signal, should be practically uncorrelated.

- $\mathrm{R}_{\mathrm{PC}}$ : should go to zero because of the negative feedback of the loop.

- $\mathrm{R}_{\mathrm{PI}}$ : is equal to $\varepsilon_{\mathrm{p}} / \mathrm{G}_{\mathrm{Loop}}$, the required correlation for minimizing the SI present in the $\mathrm{S}_{\mathrm{FR}}$ corresponding to $\mathrm{D}[\mathrm{p}]$.

The $\mathrm{R}_{\mathrm{PI}}$ acts as a control input for the negative feedback SI-cancellation loop. Additionally, $\mathrm{R}_{\mathrm{PI}}$ is also the residualSI present in $S_{F R}$, and its value depends upon the $G_{\text {Loop }}$. Therefore, $\mathrm{G}_{\text {Loop }}$ should be large for reducing the residual$\mathrm{SI}$, and to faithfully recover the far-end information from $\mathrm{S}_{\mathrm{RI}}$.
Using this correlation-based cancellation approach, this work proposes a TFD-IO cell that supports bidirectional data transfer over an electrical interconnect. The use of the TFD-IO cell supports background suppression of the interference using a correlation-based SI-canceller.

\section{Proposed True-Full Duplex IOS}

The proposed TFD-IO, as shown in Fig. 4, consists of a line-driver (LD) for signal transmission, a receiver front-end (RFE) as a buffer for signal reception, and the correlationbased SI-canceller for interference suppression. The correlative approach allows the canceller to adapt in the background for suppressing the time-variations of interference. The canceller supports interference cancellation spanning over multiple bits of the near-end transmitted data.

Figure 4 shows the architecture of correlation-based SIcanceller. The SI-canceller is used to cancel $\mathrm{S}_{\mathrm{NI}}$ present in $\mathrm{S}_{\mathrm{RI}}$, which includes the SI received from LD, and the immediate echoes/reflections from the channel-IO interface. The canceller uses the correlation-based cancellation algorithm to decide its weight coefficients $\mathrm{c}_{[1: \mathrm{k}]}$ for the delayed taps $\mathrm{D}[1: \mathrm{k}]$. The SIcanceller generates the replica tap $\mathrm{S}_{\mathrm{C}}$, as a sum of the weighted taps $T[1: \mathrm{k}]$ to suppress the interference, as given by Eq. (5),

$$
\mathrm{S}_{\mathrm{C}}=\sum_{\mathrm{p}=1}^{\mathrm{k}} \mathrm{T}[\mathrm{p}]=\sum_{\mathrm{p}=1}^{\mathrm{k}} \mathrm{c}_{\mathrm{p}} \times \mathrm{D}[\mathrm{p}] .
$$

The cancellation loop uses the coefficients $\mathrm{c}_{[1: \mathrm{k}]}$ to minimize the correlation between $\mathrm{S}_{\mathrm{FR}}$ and $\mathrm{D}[1: \mathrm{k}]$, to reduce the interference. As the canceller minimizes only the correlation, by subtracting $\mathrm{S}_{\mathrm{C}}$ from $\mathrm{S}_{\mathrm{RI}}$, it preserves the uncorrelated far-end information at the output of summer, and helps in extracting the far-end information from $S_{F R}$. Hence, the value of $c_{k}$ depends only on the correlation between $\mathrm{D}[\mathrm{k}]$ and constituents of $S_{\mathrm{NI}}$, and is independent of the far-end signal. Deviations in the value of $c_{k}$ from the actual correlation may occur due to: (i) the presence of some finite correlation between the far-end and near-end transmitted signals; (ii) DC-offsets in the cancellation loops; and (iii) the finite loop gain of the correlator, leaving a residual-SI in $\mathrm{S}_{\mathrm{FR}}$ at canceller's output. 


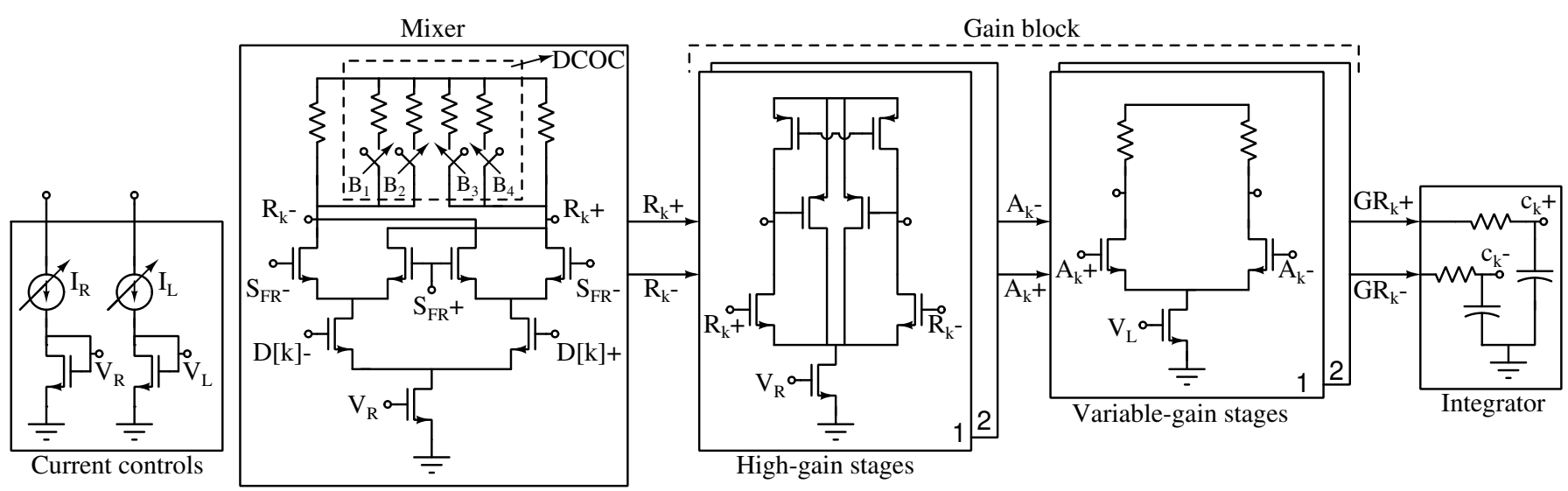

Fig. 5: Correlator logic circuit with DCOC. Here, $B_{k}: k^{\text {th }}$ control bit of DCOC.

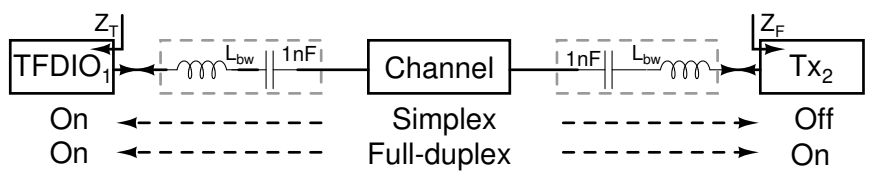

Fig. 6: Simulation setup for the TFD-IO cell.

The canceller's implementation is entirely analog using current mode logic. Each canceller tap includes a correlator and a weighting amplifier WA -implemented as a Gilbert-cell. As shown in Fig. 5, the correlator uses a mixer for determining the instantaneous correlation between a delayed tap D[k] and the summer output $\mathrm{S}_{\mathrm{FR}}$. The mixer's output is then amplified and integrated to determine $c_{k}$ using a gain block and a low pass filter, respectively. The weight coefficient $c_{k}$ controls the gain of $\mathrm{WA}_{\mathrm{k}}$ for generating the weighted tap $\mathrm{T}[\mathrm{k}]$. Depending upon the data rates and interfacing scenarios, the $c_{k}$ can have both the polarities and can take a wide range of values.

Figure 5 shows the circuit implementation of the correlator. The mixer is implemented using a differential double balanced configuration with programmable load resistances, which serves as a DC offset compensator (DCOC) to correct offset in the loop. These offsets can occur due to various system anomalies, such as process, voltage, and temperature variations. The mixer output is amplified using the gain block. The gain block is a cascade of two differential op-amps with an inbuilt common-mode feedback circuit and two differential amplifiers with variable gains. Variable gain provides additional flexibility to adjust the loop gain and maintain the stability of the loop. The output of the cascaded gain block is further integrated using the low pass filter to determine the weight coefficient $c_{k}$. The negative feedback action of the cancellation loop reduces the interference component at the output of summer. The summer is implemented using resistances to add currents from various stages of the loop. For testing purpose, the TFD-IO cell has a built-in $2^{7}-1$ pseudo-random-bit-sequence (PRBS) generator.

\section{Simulation RESUlts}

The loop gain of the canceller, far-end termination, and bondwire inductance play critical roles in the interference
TABLE I: Effect of loop gain, bondwire inductance, and far-end termination on Residual-SI

\begin{tabular}{|l|c|c|c|}
\hline $\begin{array}{l}\text { Bias current } \mathrm{I}_{\mathrm{L}}(\text { in } \mu \mathrm{A}) \\
\mathrm{L}_{\mathrm{bw}}=1 \mathrm{nH}, \mathrm{Z}_{\mathrm{F}}=50\end{array}$ & 40 & 70 & 100 \\
\hline Residual-SI (in $\left.\mathrm{mV}_{\mathrm{rms}}\right)$ & 9.47 & 6.78 & 6.75 \\
\hline \hline $\begin{array}{l}\text { Far-end termination } \mathrm{R}_{\mathrm{F}}(\Omega) \\
\mathrm{L}_{\mathrm{bw}}=1 \mathrm{nH}, \mathrm{I}_{\mathrm{L}}=100 \mu \mathrm{A}\end{array}$ & 45 & 50 & 55 \\
\hline Residual-SI (in $\mathrm{mV}_{\mathrm{rms}}$ ) & 6.5 & 6.7 & 7.0 \\
\hline \hline $\begin{array}{l}\text { Bondwire inductance } \mathrm{L}_{\mathrm{bw}}(\text { in } \mathrm{nH}) \\
\mathrm{R}_{\mathrm{F}}=50 \Omega, \mathrm{I}_{\mathrm{L}}=100 \mu \mathrm{A}\end{array}$ & 0.5 & 1.0 & 1.5 \\
\hline Residual-SI (in $\left.\mathrm{m}_{\mathrm{rms}}\right)$ & 4.8 & 6.7 & 8.2 \\
\hline
\end{tabular}

cancellation process. Post-layout simulations are performed to analyze the effect of the above three on the residual-SI. The simulation setup as shown in Fig. 6, includes an inductor $\mathrm{L}_{\mathrm{bw}}$ and a $1 \mathrm{nF}$ capacitor, at each communicating end for emulating a bondwire inductance and an ac-coupling capacitance, respectively. A channel with a characteristic impedance of $50 \Omega$ and a loss of $6 \mathrm{~dB}$ at the Nyquist frequency is used for the simulations. All simulations are performed in the TypicalTypical corner at $100^{\circ} \mathrm{C}$. For simulations, the built-in $2^{7}-1$ PRBS generator of TFD- $\mathrm{IO}_{1}$ is used as the near-end source and an uncorrelated $2^{31}-1$ PRBS is transmitted from the far-end source $\mathrm{Tx}_{2}$.

Table I shows the effect of bias current $\mathrm{I}_{\mathrm{L}}$, which controls the loop gain on the residual-SI. There is an inverse relation between residual-SI and the loop gain, which is consistent with the analytical analysis in Section II. Table I shows an increase in residual-SI with an increase in $\mathrm{L}_{\mathrm{bw}}$. This increase is because of higher reflections at the near-end as the bondwire inductance $\mathrm{L}_{\mathrm{bw}}$ deteriorates the reflection parameter $S_{11}$. The effect of impedance mismatch at the far-end is observed on the residual-SI by varying $\mathrm{R}_{\mathrm{F}}$, and is shown in Table I. Channel loss and proper impedance matching at the far-end significantly reduce the unwanted reflections received at the near-end in wire-line communication.

\section{Measurement SETUP AND ExPERIMENTAl RESUlts}

A prototype of the proposed TFD-IO is designed, and fabricated in a standard $65-\mathrm{nm}$ CMOS low-leakage process with 


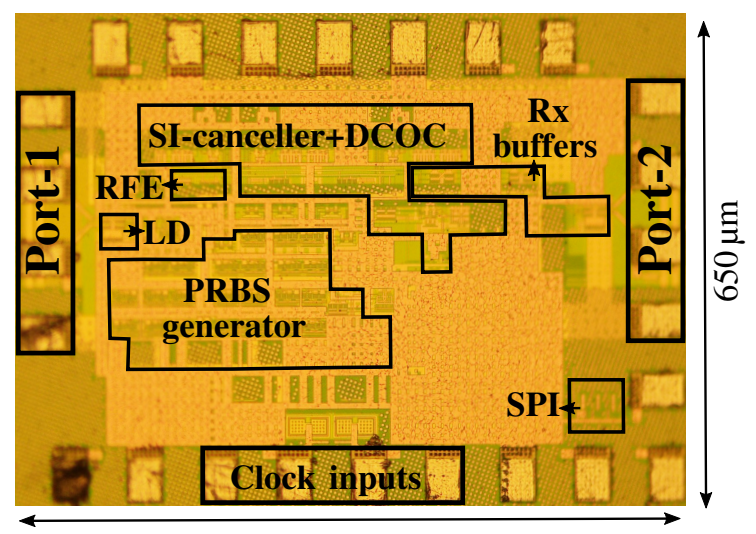

$900 \mu \mathrm{m}$

Fig. 7: Die-micrograph of TFD-IO.

a supply voltage of $1.2 \mathrm{~V}$. Fig. 7 shows the die micrograph of TFD-IO. The active core of TFD-IO has an area of $\approx 0.1 \mathrm{~mm}^{2}$. For the measurements, a chip-on-board $(\mathrm{CoB})$ arrangement is used with both the DC and the RF pads wire-bonded to a four-layer FR-4 PCB. PCB traces are designed to have a differential impedance of $100 \Omega$ for high-speed data and clock signals. Fig. 8 shows the experimental setup along with the channel response. The channel has a transmission loss $\left(\mathrm{S}_{21}\right)$ of $-8 \mathrm{~dB}$ at $3.2 \mathrm{GHz}$ and return losses $\left(\mathrm{S}_{11}\right)$ better than $-10 \mathrm{~dB}$ upto $3.7 \mathrm{GHz}$. TFD-IO is AC-coupled at port-1 using $10 \mathrm{nF}$ capacitors to isolate DCs of the two ends.

DCOC is manually programmed to perform a coarse correction for DC-offsets present in the SI-canceller. DCOC is initialized using an on-chip serial-to-parallel interface (SPI) with the correction word required to correct for the offsets, which may arise due to variations in the process, voltage, and temperature (PVT). The near-end and far-end data are generated using an on-chip $2^{7}-1$ PRBS generator and an external arbitrary waveform generator (A4), respectively. Our proposed TFD-IO is configured to transmit and receive signals with differential swings of $400 \mathrm{mV}_{\mathrm{pp}}$. The performances of TFDIO's transmitter, receiver, and correlation-based SI-canceller are verified as follows:

Transmitter: The system configuration utilized for the measurements is shown in Fig. 9(a). TFD-IO is used to generate the near-end signal and the far-end source is configured as a $100 \Omega$ differential load. The near-end signal, as a result of TFD-IO's bidirectional architecture, appears as $\mathrm{S}_{\mathrm{TR}}$ and $\mathrm{S}_{\mathrm{FR}}$, at port-1 and port-2, respectively. Here, $\mathrm{S}_{\mathrm{TR}}$ is the desired near-end transmitted signal, whereas $S_{\mathrm{FR}}$ is the undesired SI. For $\mathrm{S}_{\mathrm{FR}}$, the eye-diagram is plotted in Fig. 10(a), which shows a differential swing of approximately $440 \mathrm{mV}_{\mathrm{pp}}$ for the interference.

Receiver: To verify the performance of TFD-IO as a receiver, the PRBS generator as well SI-canceller are deactivated and the far-end data is transmitted from the arbitrary waveform generator, as shown in Fig. 9(c). Fig. 11(a) shows the far-end signal received at port-2. The received far-end signal has a differential swing of approximately $440 \mathrm{mV}_{\mathrm{pp}}$. TFD-IO shows similar swings for the SI and the received far-end signal,

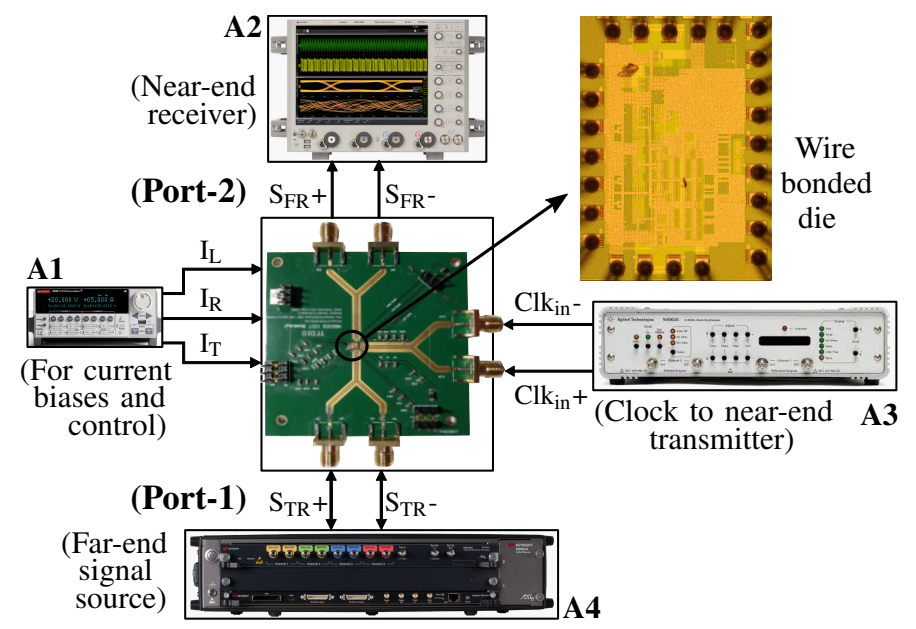

(a)

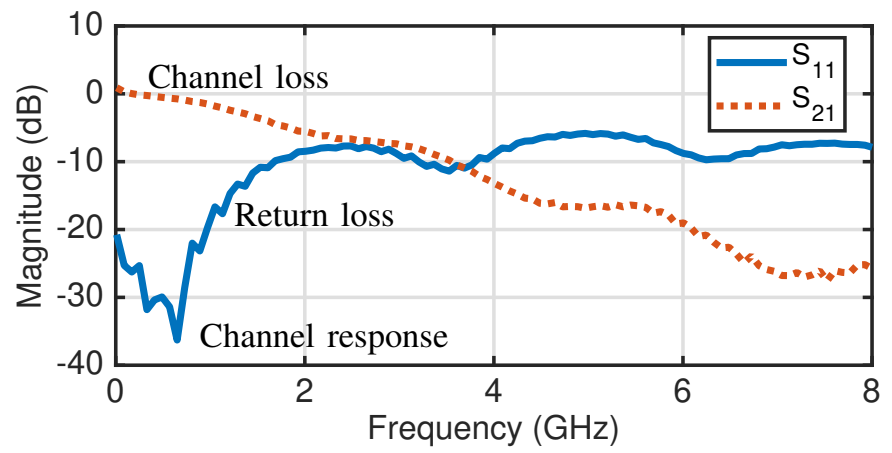

(b)

Fig. 8: (a) Experimental setup and (b) the channel response. Here, $\mathrm{I}_{\mathrm{T}}$ : control for the output swing of near-end transmitter; $\mathrm{I}_{\mathrm{R}}$ : control for the linearity of near-end RFE; $\mathrm{I}_{\mathrm{L}}$ : control for loop gain of SIcanceller; A1: source meters; A2: digital storage oscilloscope; A3: frequency synthesizer; and A4: arbitrary waveform generator.

as plotted in Fig. 10(a) and Fig. 11(a), respectively. They are similar because the Rx buffer has a gain at low frequencies, which limits or saturates the output swing at port-2.

In the above two configurations, TFD-IO can reliably transmit and receive data without any processing over simplex links but need interference suppression for full-duplex operations.

SI-canceller: TFD-IO uses the correlation-based SIcanceller to suppress the interference, in the analog domain, at the output of the summer. To validate canceller's performance, near-end transmitter along with the SI-canceller is active, and the far-end source is configured as a $100 \Omega$ differential load, as shown in Fig. 9(b). Fig. 10(b) and Fig. 10(c) show the residualSI present in $\mathrm{S}_{\mathrm{FR}}$ at port-2, post interference cancellation, and post interference cancellation plus DC offset correction, respectively. The cancellation along with offset correction reduces the residual-SI from $163 \mathrm{mV}_{\text {rms }}$ to $30 \mathrm{mV}_{\text {rms }}$, and thus provides an interference suppression of $14.7 \mathrm{~dB}$.

Interference suppression performed using the proposed SIcanceller is required to recover far-end information in TFD links. As discussed in Section II, the correlation-based approach for interference cancellation allows the SI-canceller to adapt its tap values in the background, independent of the far- 


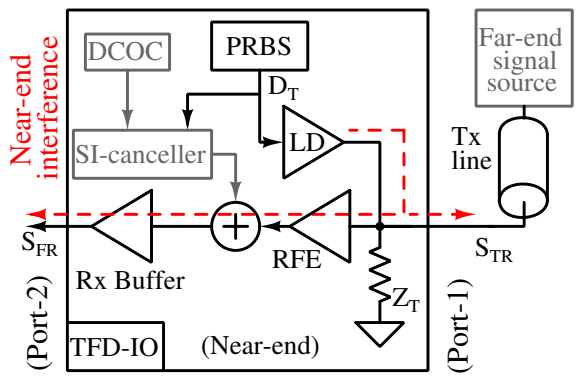

(a)

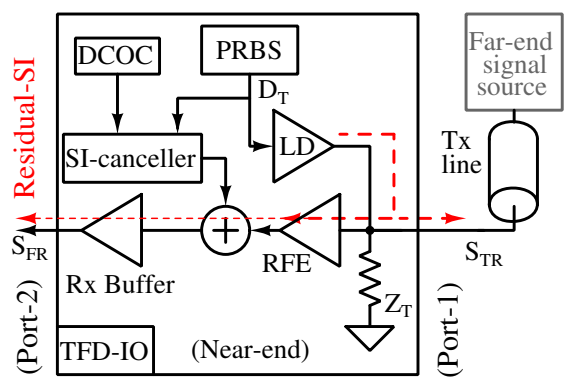

(b)

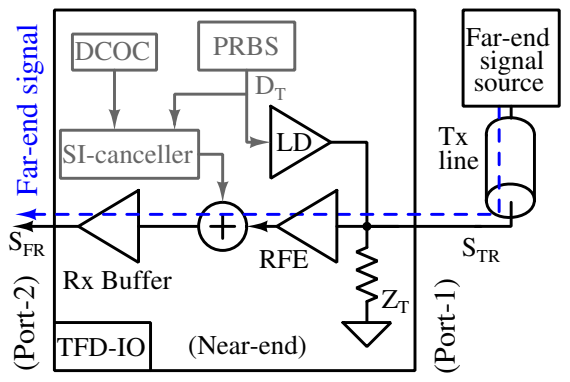

(c)

Fig. 9: Configurations of TFD-IO to verify the performance of its: (a) transmitter, (b) SI-canceller, and (c) receiver. Inactive components and connections are shown using gray color. Dashed lines show path followed by the signals.

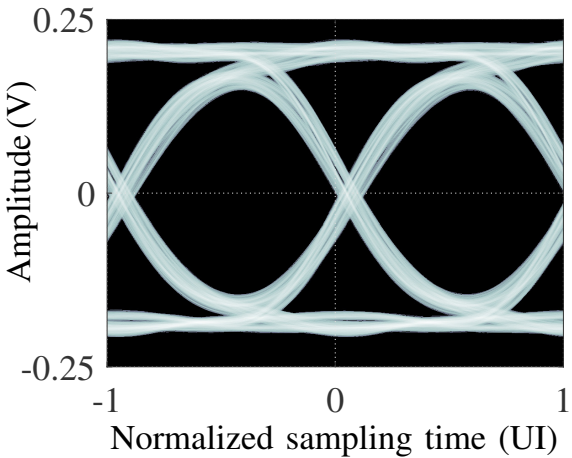

(a)

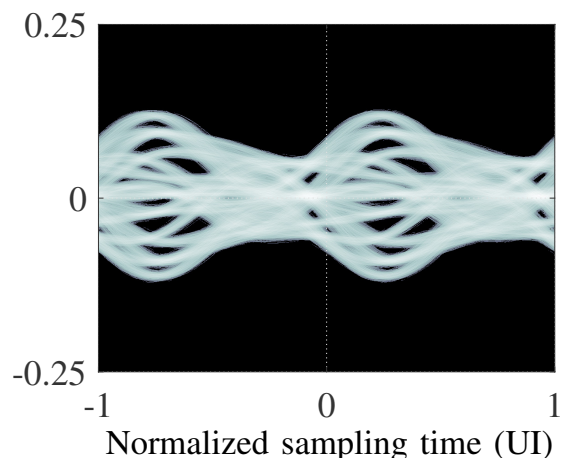

(b)

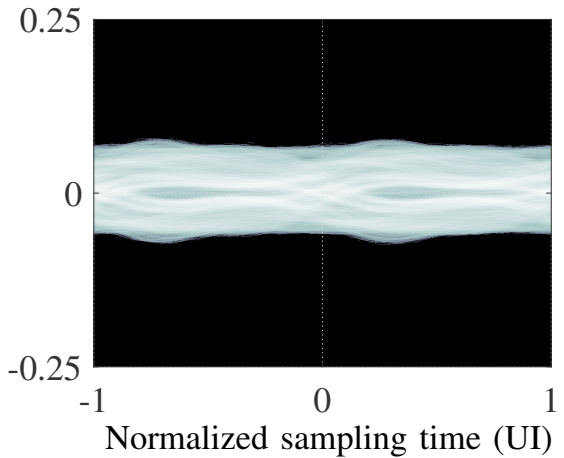

(c)

Fig. 10: Eye diagram plotted for $20 \mathrm{k}$ symbols of $S_{F R}$ with the near-end transmitter active and the far-end source inactive: (a) Without interference cancellation, (b) With interference cancellation, and (c) With interference cancellation and offset correction. These measurements were performed with the TFD-IO operating at $6.4 \mathrm{~Gb} / \mathrm{s}$.

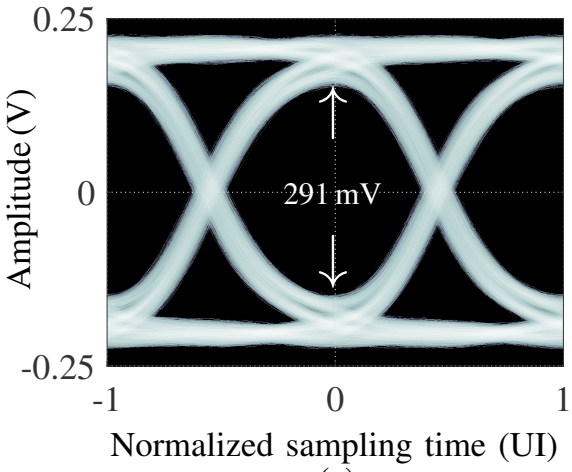

(a)

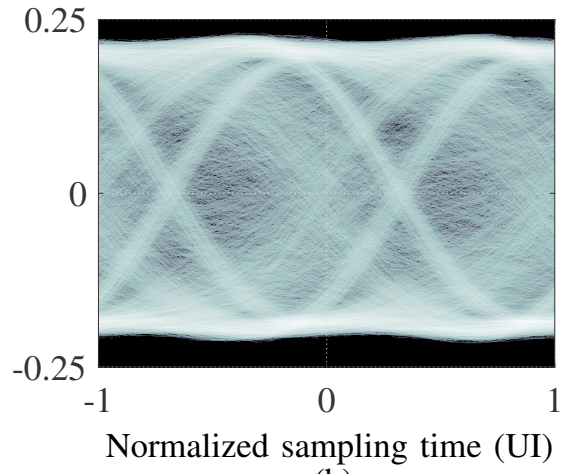

(b)

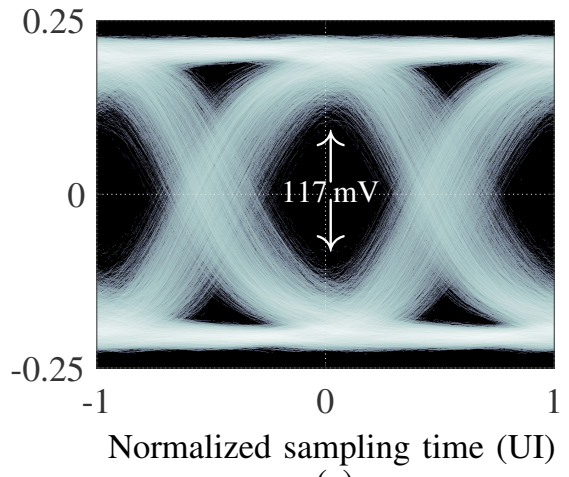

(c)

Fig. 11: Eye diagram plotted for $20 \mathrm{k}$ symbols of $S_{\mathrm{FR}}$ with the far-end source active: (a) Without near-end interference, (b) With nearend interference, and (c) With interference cancellation and DC offset correction. These measurements were performed with the two ends operating at $6.4 \mathrm{Gpbs}$, the link thus supports an aggregate bidirectional throughput of $12.8 \mathrm{~Gb} / \mathrm{s}$.

end signal. The TFD-IO is operated in the following system configurations to validate the concept of true full-duplexing.

\section{A. Same baud-rates and signalling schemes}

The measurements are performed for a full-duplex link with the two ends, simultaneously transmitting, at data rates of 6.4 Gb/s using NRZ signalling scheme. Fig. 11(b) shows eyediagrams of the composite signal received at port-2, which comprises the far-end signal and the near-end interference. The presence of interference closes the eye of far-end signal as shown in Fig. 11(b) as compared to Fig. 11(a), where near-end interference is absent.
Conventionally, to extract the far-end information, adaptive interference cancellers in such links may require a foreground training and a phase locked arrangements [19], [20], [30], [31]. Whereas, the proposed TFD-IO because of the correlationbased SI-canceller can adapt its weights in presence of the farend signal. Fig. 11(c) shows eye-diagram of the far-end signal recovered post interference cancellation. The eye-diagrams show a reduction in the eye-height and eye-width because of the presence of residual-SI.

\section{B. Different baud-rates and same signalling schemes}

To validate the ability of SI-canceller to support interference cancellation independent of the phase of far-end signal, the 


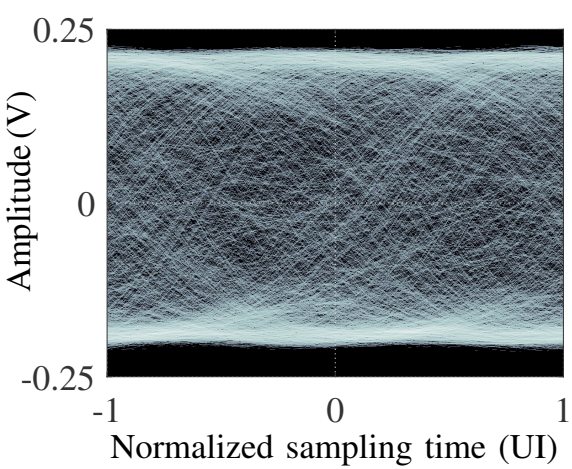

(a)

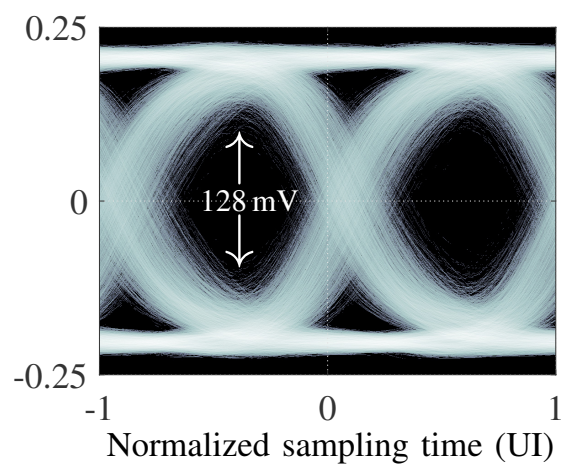

(b)

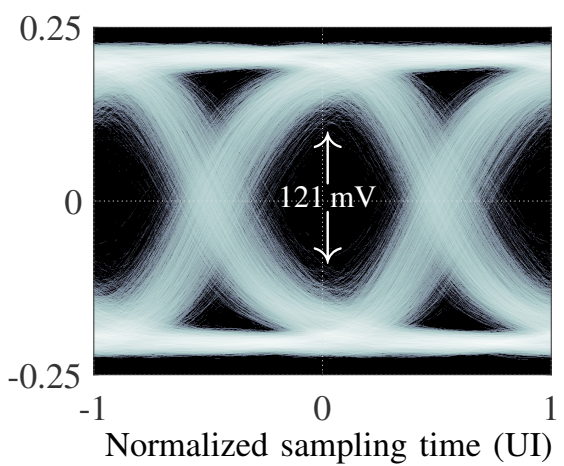

(c)

Fig. 12: Eye diagram plotted for 20k symbols of $S_{F R}$ in TFD mode: (a) received far-end signal without interference cancellation, (b) recovered far-end signal $(6.25 \mathrm{~Gb} / \mathrm{s})$ with interference cancellation plus offset correction, and (c) recovered far-end signal $(6.4 \mathrm{~Gb} / \mathrm{s})$ with interference cancellation plus offset correction. These measurements were performed with the two ends operating at different baud rates.

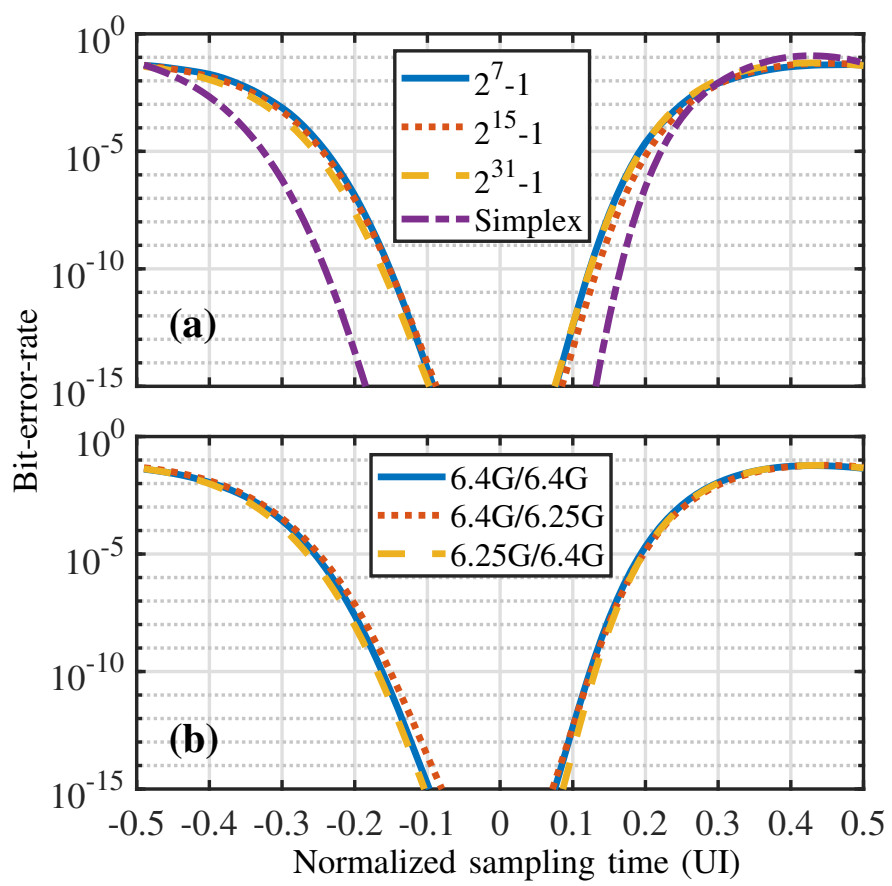

Fig. 13: Bath-tub curves to estimate BER for: (a) the near-end (simplex) and the recovered far-end (transmitted using different PRBS sequences) signals operated at $6.4 \mathrm{~Gb} / \mathrm{s}$, and (b) the recovered far-end signal with the two ends operating at different baud rates.

measurements are performed with the two ends operating at different baud-rates using same NRZ signalling scheme. Fig. 12(a) shows eye-diagram of the composite signal received at port-2, which comprises the far-end signal and the interference due to near-end, operating at $6.25 \mathrm{~Gb} / \mathrm{s}$ and $6.4 \mathrm{~Gb} / \mathrm{s}$, respectively. In such an operating condition, the relative phase of the two ends continuously varies. The interference, thus completely closes the eye-opening of far-end signal. SIcanceller is used to suppress the interference for recovering the far-end signal. Fig. 12(b) shows eye-diagram of the recovered far-end signal. In addition, Fig. 12(c) shows eye-diagram of the recovered signal, for the measurement, where the farend and the near-end are operated at $6.4 \mathrm{~Gb} / \mathrm{s}$ and $6.25 \mathrm{~Gb} / \mathrm{s}$, respectively. Both eye-diagrams show a reduction in the eyewidth and eye-height similar to Fig. 11(c).

Bath-tub curves are used to estimate the bit-error-rates
(BER)s for the far-end signal recovered at port-2. The curves are plotted using Eq. (6) as given in [34], by determining the signal-to-noise ratio (SNR) at each sample point.

$$
\mathrm{BER}=\frac{1}{2} \times Q(\sqrt{\mathrm{SNR}}) .
$$

Figure 13(a) shows bath-tub curves for the recovered signal corresponding to three PRBS sequences (used for transmitting the far-end data): $2^{7}-1,2^{15}-1$, and $2^{31}-1$. These curves show a similar BERs, and thus advise a nearby signalto-interference ratio for the recovered signals. In addition, Fig. 13(a) also shows BER for the near-end transmitted signal, in simplex mode, as received at port-2. Here, correlation-based SI-canceller adapts its weights in the background independent of the data sequence (or information) used for transmitting the far-end signal.

Figure 13(b) shows the estimated bath-tub curves with the two ends operating at the same and different baud-rates. These curves are plotted for the PRBS sequence $2^{31}-1$ used for transmitting the far-end data. The curves are for the following three combinations of near-end and far-end baud-rates: (i) $6.4 \mathrm{~Gb} / \mathrm{s}$ (phase of near-end and far-end data change at the same rate), (ii) $6.4 \mathrm{~Gb} / \mathrm{s} / 6.25 \mathrm{~Gb} / \mathrm{s}$ (phase of near-end data changes faster than the far-end data), and (iii) $6.25 \mathrm{~Gb} / \mathrm{s} / 6.4 \mathrm{~Gb} / \mathrm{s}$ (phase of far-end data changes faster than the near-end data). The bathtub curves for the three combinations show a similar error rate for the recovered far-end signal, and thus verify the ability of SI-canceller to support interference cancellation independent of the phase or baud-rate of far-end data.

\section{Same baud-rates and different signalling schemes}

To validate SI-canceller's ability to support interference cancellation at the near-end, independent of the signalling scheme used by far-end transceiver, a proof of concept demonstration is done with different signalling schemes used at the two ends. The near-end and far-end signals are transmitted at $500 \mathrm{MBauds}$ per second using NRZ and PAM4 signalling schemes, respectively. To operate in the linear region, the gain of the Rx buffers is lowered using $I_{R}$, and the output swing of the near-end transmitted signal is reduced to $80 \mathrm{mVpp}$ using $\mathrm{I}_{\mathrm{T}}$. Fig. 14(a) and Fig. 14(b) show eye-diagrams of the far-end PAM4 signals received without and with near-end interference, respectively. In contrast to Fig. 14(a), the eyes for $S_{F R}$ in 


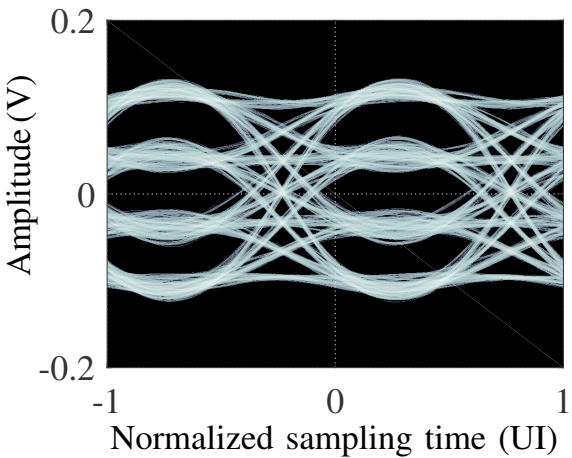

(a)

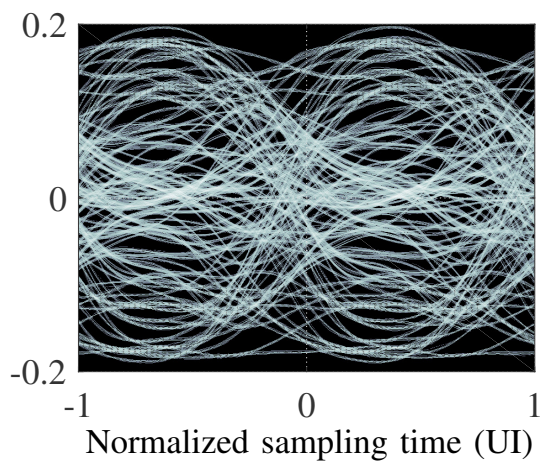

(b)

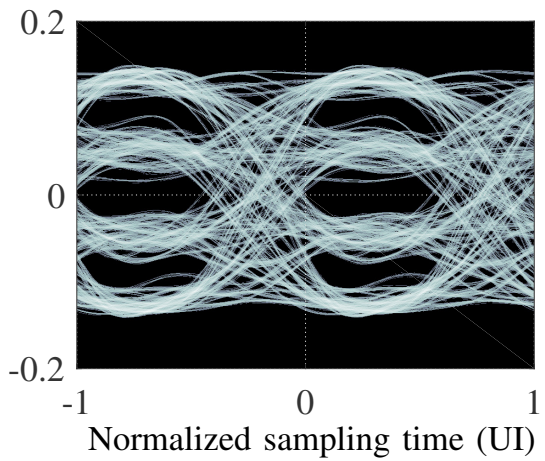

(c)

Fig. 14: Eye diagram plotted for $5 \mathrm{k}$ symbols of $\mathrm{S}_{\mathrm{FR}}$ in TFD mode: (a) received far-end PAM4 signal without near-end interference, (b) received far-end PAM4 signal without interference cancellation, and (c) recovered far-end PAM4 signal with interference cancellation plus offset correction. These measurements were performed with NRZ and PAM4 used as the near-end and far-end signalling schemes, respectively.

TABLE II: PERFORMANCE SUMMARY AND COMPARISON TO PRIOR ART

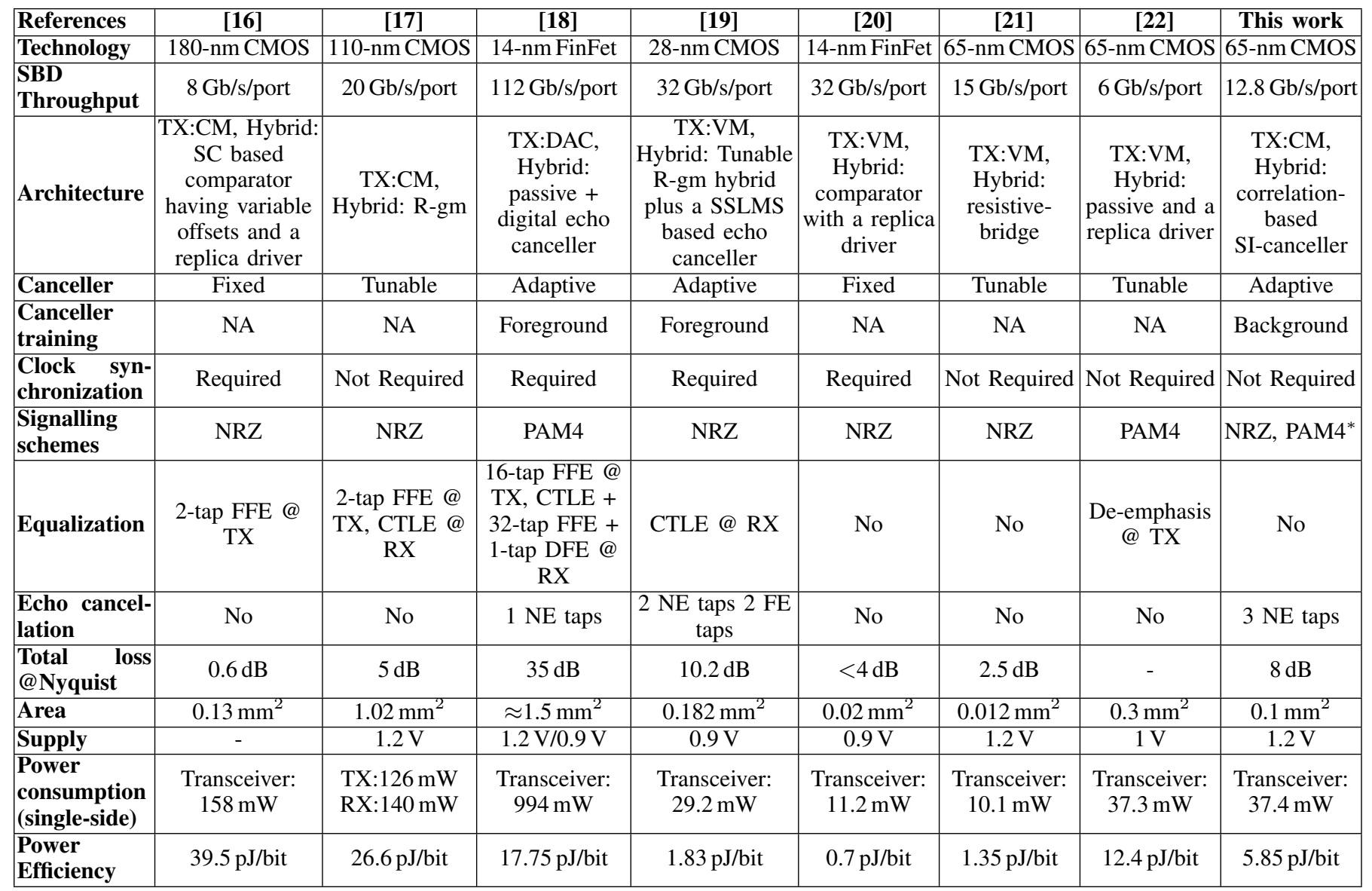

*-Supported by the SI-canceller and Rx.

Fig. 14(b) are entirely closed. Fig. 14(c) shows eye-diagram of the far-end PAM4 signal received post near-end interference suppression performed using SI-canceller. Fig. 14(c) shows a significant eye opening for the received signal as compared to Fig. 14(b). Thus, the SI-canceller can faithfully recover the far-end PAM4 signal while using NRZ signalling scheme for near-end data transmission.

In the above configurations, the far-end information was recovered with the SI-canceller operated in the background. These measurements, thus validate SI-canceller's ability to adapt in the presence of far-end signal, independent of the sig- nalling schemes and baud rates used by the transceivers at the two ends. TFD-IO with the correlation-based SI-canceller, thus eliminates the need to have a phase/frequency synchronization or clock forwarding between the two transceivers. Additionally, TFD-IO can also support interference cancellation in reconfigurable transceivers [35], [36] that allow data transfers in multiple signalling schemes. A summary of the TFD-IO performance, and its comparison to prior-art transceivers is given in Table II. The demonstrated TFD link supports an aggregate bidirectional data throughput of $12.8 \mathrm{~Gb} / \mathrm{s}$, with a BER better than $10^{-9}$ for a sampling window of $0.3 \mathrm{UI}$. 


\section{CONCLUSION}

In this work, we presented a true full-duplex IO (TFD-IO) that can serve as a plug-n-play module for high-density interfaces to convert a unidirectional interconnect to a fully bidirectional interconnect. The TFD-IO performs interference suppression in the analog domain using a correlation-based SI-canceller to support bidirectional data transfer. The SI-canceller adaptively determines its coefficients even in the presence the signal transmitted by the far-end transceiver. TFD-IO has been experimentally validated with a proof-of-concept demonstration for a bidirectional throughput of up to $12.8 \mathrm{~Gb} / \mathrm{s}$. The interference cancellation technique used in the TFD-IO allows for the use of different baud-rates and signalling schemes, such as NRZ/PAM4, by the two transceivers. The IO module presented in this work can be used in a practical design for doubling the throughput of the interface. This approach can be very useful in improving the data bandwidth of a high-density interface. The TFD-IOs should also help in simplifying the floor planning of PCBs for chip-to-chip multi-lane high-speed interfaces, as well as for interfaces in chiplet based designs used in SiPs/MCMs.

\section{ACKNOWLEDGEMENT}

The authors thank the SMDP-C2SD program of MeitY (Govt. of India), and Qualcomm Innovation Fellowship, for supporting this activity in parts. We would also like to acknowledge Mr. Mukund Narasimhan and Mr. Shajith Sirajudeen from Qualcomm, for some useful discussions. Initial funding by SRC for activity in this area (task no. 2739.001) is also acknowledged.

\section{REFERENCES}

[1] F. O’Mahony, G. Balamurugan, J. E. Jaussi, J. Kennedy, M. Mansuri, S. Shekhar, and B. Casper, "The future of electrical I/O for microprocessors," in IEEE Symp. on VLSI Design, Automation and Test, 2009, pp. 31-34

[2] J. Macri, "AMD's next generation GPU and high bandwidth memory architecture: FURY," in IEEE Hot Chips, 2015, pp. 1-26.

[3] Y. Wen, "The package bandwidth limitation of high speed broadband products," in IEEE ECTC, 2001, pp. 1457-1462.

[4] J. W. Poulton, W. J. Dally, X. Chen, J. G. Eyles, T. H. Greer, S. G. Tell, and C. T. Gray, "A $0.54 \mathrm{pJ} / \mathrm{b} 20 \mathrm{~Gb} / \mathrm{s}$ ground-referenced singleended short-haul serial link in $28 \mathrm{~nm}$ CMOS for advanced packaging applications," in IEEE ISSCC Dig. Tech. Papers, 2013, pp. 404-405.

[5] A. C. Durgun, Z. Qian, K. Aygun, R. Mahajan, T. T. Hoang, and S. Y. Shumarayev, "Electrical Performance Limits of Fine Pitch Interconnects for Heterogeneous Integration," in IEEE ECTC, 2019, pp. 667-673.

[6] A. Shokrollahi, D. Carnelli, J. Fox, K. Hofstra, B. Holden, A. Hormati, P. Hunt, M. Johnston, J. Keay, S. Pesenti, R. Simpson, D. Stauffer, A. Stewart, G. Surace, A. Tajalli, O. T. Amiri, A. Tschank, R. Ulrich, C. Walter, F. Licciardello, Y. Mogentale, and A. Singh, "A pin-efficient $20.83 \mathrm{~Gb} / \mathrm{s} /$ wire $0.94 \mathrm{pJ} /$ bit forwarded clock CNRZ-5-coded SerDes up to $12 \mathrm{~mm}$ for MCM packages in $28 \mathrm{~nm}$ CMOS," in IEEE ISSCC, 2016, pp. $182-183$.

[7] D. S. Green, "Common Heterogeneous Integration and Intellectual Property (IP) Reuse Strategies (CHIPS)," [Online]. Available: https://www.darpa.mil/attachments/CHIPSoverview\% 20Sept212016ProposerDay.pdf [Accessed: 20-08-2020].

[8] D. Kehlet, "Accelerating Innovation Through a Standard Chiplet Interface: The Advanced Interface Bus," [Online]. Available: https://www.intel.com/content/dam/www/public/us/en/documents/ white-papers/accelerating-innovation-through-aib-whitepaper.pdf [Accessed: 20-08-2020].
[9] J. Fourniols, M. Roca, F. Caignet, and E. Sicard, "Characterization of crosstalk noise in submicron CMOS integrated circuits: an experimental view," IEEE Trans. Electromagn. Compat., vol. 40, no. 3, pp. 271-280, 1998.

[10] F. D. Mbairi, W. P. Siebert, and H. Hesselbom, "High-Frequency Transmission Lines Crosstalk Reduction Using Spacing Rules," IEEE Trans. Compon. Packag. Technol., vol. 31, no. 3, pp. 601-610, 2008.

[11] P. Roo, S. Sutardja, S. Wei, F. Aram, and Y. Cheng, "A CMOS transceiver analog front-end for gigabit ethernet over CAT-5 cables," in IEEE ISSCC Dig. Tech. Papers., 2001, pp. 310-311.

[12] “IEEE 802.3 Ethernet Working Group," [Online]. Available: http://www. ieee802.org/3 [Accessed: 20-08-2020].

[13] G. Chandra and M. Malkin, "A full-duplex 10GBase-T transmitter hybrid with SFDR $>65 \mathrm{dBc}$ Over 1 to $400 \mathrm{MHz}$ in $40 \mathrm{~nm}$ CMOS," in IEEE ISSCC, 2011, pp. 144-146.

[14] J. Chen, Y. Gu, and K. K. Parhi, "Low-Complexity Echo and NEXT Cancellers for High-Speed Ethernet Transceivers," IEEE TCASI: Regular Papers, vol. 55, no. 9, pp. 2827-2840, 2008.

[15] S. Sen, F. Aryanfar, and C. Werner, "A Multiband Transceiver System in 45-nm CMOS for Extended Data Rate through Notchy Wireline Channels," IEEE TCASII: Express Briefs, vol. 58, no. 9, pp. 545-549, 2011.

[16] B. Casper, A. Martin, J. Jaussi, J. Kennedy, and R. Mooney, “An 8 Gb/s simultaneous bidirectional link with on-die waveform capture," IEEE J. Solid-State Circuits, vol. 38, no. 12, pp. 2111-2120, 2003.

[17] Y. Tomita, H. Tamura, M. Kibune, J. Ogawa, K. Gotoh, and T. Kuroda, "A $20 \mathrm{~Gb} / \mathrm{s}$ Simultaneous Bidirectional Transceiver Using a ResistorTransconductor Hybrid in 0.11- $\mu \mathrm{m}$ CMOS," IEEE J. Solid-State Circuits, vol. 42, no. 3, pp. 627-636, 2007.

[18] R. Farjadrad, K. Kaviani, D. Nguyen, M. Brown, G. Geelen, C. Bastiaansen, N. Rao, V. Popuri, G. Shen, H. Khatibi, S. Dey, A. Chatterjee, D. Shen, P. Zijlstra, H. Gunnink, K. Zhang, V. Penumuchu, O. Weiss, E. Paulus, and J. Briaire, "An Echo-Cancelling Front-End for 112Gb/s PAM-4 Simultaneous Bidirectional Signaling in 14nm CMOS," in IEEE ISSCC, vol. 64, 2021, pp. 194-196.

[19] Y.-H. Fan, A. Kumar, T. Iwai, A. Roshan-Zamir, S. Cai, B. Sun, and S. Palermo, "A $32 \mathrm{~Gb} / \mathrm{s}$ Simultaneous Bidirectional Source-Synchronous Transceiver With Adaptive Echo Cancellation Techniques," IEEE $J$. Solid-State Circuits, vol. 55, no. 2, pp. 439-451, 2020.

[20] R. Farjadrad, M. Kuemerle, and B. Vinnakota, "A Bunch-of-Wires (BoW) Interface for Interchiplet Communication," IEEE Micro, vol. 40, no. 1, pp. 15-24, 2020.

[21] C. Yuan, A. Naguib, and S. Shekhar, "On the Design of Low-Power Hybrids for Full Duplex Simultaneous Bidirectional Signaling Links," IEEE TCASI: Regular Papers, vol. 67, no. 4, pp. 1413-1422, 2020.

[22] S. Mukherjee, A. Das, S. Seth, and S. Saxena, "An Energy-Efficient $3 \mathrm{~Gb} / \mathrm{s}$ PAM4 Full-Duplex Transmitter With 2-Tap Feed Forward Equalizer," IEEE TCASII: Express Briefs, vol. 67, no. 5, pp. 916-920, 2020.

[23] H. Wilson and M. Haycock, "A six-port $30 \mathrm{~Gb} / \mathrm{s}$ nonblocking router component using point-to-point simultaneous bidirectional signaling for high-bandwidth interconnects," IEEE J. Solid-State Circuits, vol. 36, no. 12 , pp. 1954-1963, 2001.

[24] R. Drost and B. Wooley, "An $8 \mathrm{~Gb} / \mathrm{s} /$ pin simultaneously bidirectional transceiver in 0.35-/mum CMOS," IEEE J. Solid-State Circuits, vol. 39, no. 11, pp. 1894-1908, 2004

[25] J.-H. Kim, S. Kim, W.-S. Kim, J.-H. Choi, H.-S. Hwang, C. Kim, and S. Kim, "A 4- Gb/s/pin low-power memory I/O interface using 4level simultaneous bi-directional signaling," IEEE J. Solid-State Circuits, vol. 40, no. 1, pp. 89-101, 2005.

[26] M. T. L. Aung, E. Lim, T. Yoshikawa, and T. T. Kim, "A 3- Gb/s/ch Simultaneous Bidirectional Capacitive Coupling Transceiver for 3DICs," IEEE TCASII: Express Briefs, vol. 61, no. 9, pp. 706-710, 2014.

[27] M. Jalalifar and G. Byun, "An Energy-Efficient Mobile Memory I/O Interface Using Simultaneous Bidirectional Multilevel Dual-Band Signaling," IEEE TCASII: Express Briefs, vol. 64, no. 8, pp. 897-901, 2017.

[28] T. Kim, S. Jang, S. Kim, S. Chu, J. Park, and D. Jeong, "A Four-Channel 32- Gb/s Transceiver With Current-Recycling Output Driver and OnChip AC Coupling in 65-nm CMOS Process," IEEE TCASII: Express Briefs, vol. 61, no. 5, pp. 304-308, 2014.

[29] N. Wary and P. Mandal, "Current-Mode Full-Duplex Transceiver for Lossy On-Chip Global Interconnects," IEEE J. Solid-State Circuits, vol. 52, no. 8, pp. 2026-2037, 2017.

[30] H. Tamura, M. Kibune, Y. Takahashi, Y. Doi, T. Chiba, H. Higashi, H. Takauchi, H. Ishida, and K. Gotoh, " $5 \mathrm{~Gb} / \mathrm{s}$ bidirectional balancedline link compliant with plesiochronous clocking," in IEEE ISSCC Dig. Tech. Papers, 2001, pp. 64-65. 
[31] T. Takahashi, T. Muto, Y. Shirai, F. Shirotori, Y. Takada, A. Yamagiwa, A. Nishida, A. Hotta, and T. Kiyuna, " $110 \mathrm{~Gb} / \mathrm{s}$ simultaneous bidirectional transceiver logic synchronized with a system clock," IEEE $J$. Solid-State Circuits, vol. 34, no. 11, pp. 1526-1533, 1999.

[32] S. Goyal, P. Agarwal, and S. Gupta, "Demonstration of a Single-Lane 80 Gbps PAM-4 Full-Duplex Serial Link," in IEEE Hot Interconnects, 2019, pp. 32-35.

[33] S. Goyal, G. A. Parulekar, and S. Gupta, "A True Full-Duplex IO for High-Density High-Speed Interconnects," in IEEE ICECS, 2020, pp. 14.

[34] S. Haykin, Communication Systems, 5th ed. Wiley Publishing, 2009.

[35] A. Roshan-Zamir, O. Elhadidy, H.-W. Yang, and S. Palermo, "A Reconfigurable 16/32 Gb/s Dual-Mode NRZ/PAM4 SerDes in $65 \mathrm{~nm}$ CMOS," IEEE J. Solid-State Circuits, vol. 52, no. 9, pp. 2430-2447, 2017.

[36] V. Stojanovic, A. Ho, B. Garlepp, F. Chen, J. Wei, G. Tsang, E. Alon, R. Kollipara, C. Werner, J. Zerbe, and M. Horowitz, "Autonomous dualmode (PAM2/4) serial link transceiver with adaptive equalization and data recovery," IEEE J. Solid-State Circuits, vol. 40, no. 4, pp. 10121026, 2005. 